\title{
A IMPORTÂNCIA DO NUTRICIONISTA NA ATENÇÃO PRIMÁRIA NA PREVENÇÃO E TRATAMENTO DAS DOENÇAS CRÔNICAS NÃO TRANSMISSÍVEIS
}

\author{
Edlaine Alves da Silva ${ }^{1}$ \\ Hellba Karts Maria e Silva ${ }^{2}$ \\ Bruna Teodoro dos Santos ${ }^{3}$ \\ Edivan da Silva Gomes ${ }^{4}$ \\ Joyce Oliveira de Carvalho ${ }^{5}$
}

RESUMO: Dentre os profissionais atuantes na Atenção Básica, destaca-se a importância da inserção do nutricionista, uma vez que o enfoque do modelo de saúde proposto pelo governo não é simplesmente de recuperação da saúde e tratamento da doença, mas, principalmente, de ações voltadas para a promoção da saúde e prevenção de agravos. Dessa forma, o presente estudo tem como objetivo identificar a Importância do Nutricionista na Atenção Primária na Prevenção e Tratamento das DCNT, contribuindo com mais informações para sociedade no planejamento e execução de ações de educação alimentar e nutricional. Trata-se de uma revisão de literatura narrativa, onde foram utilizados artigos científicos que abordavam a prevenção e tratamento das doenças crônicas não transmissíveis na atenção primária em todo território brasileiro e a importância do profissional nutricionista. Ao longo dos últimos anos a alta prevalência da DCNT é um problema global e que o seu controle tem sido um verdadeiro desafio. Como exemplo desse esforço para contenção do avanço das DCNT temos desde o ano de 2011 , lançado pelo ministério da saúde o plano de ações estratégicas ,para o enfrentamento das DCNT no Brasil,20II-2022. Concluímos que a falta do nutricionista acarreta em assistência incompleta e superficial, já que a alimentação se trata de um fator biopsicossocial.

Palavra- chave: Atenção primária. DCNT. Nutricionista

\section{INTRODUÇÃO}

O estilo de vida sedentário e a mudança nos hábitos alimentares da população são os principais fatores que justificam os cenários mundial e brasileiro obesogênicos, com tendências cada vez mais alarmantes entre jovens, já que a ocorrência de excesso de peso

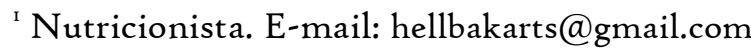

${ }^{2}$ Nutricionista

${ }^{3}$ Nutricionista

${ }^{4}$ Nutricionista

${ }^{5}$ Nutricionista
} 


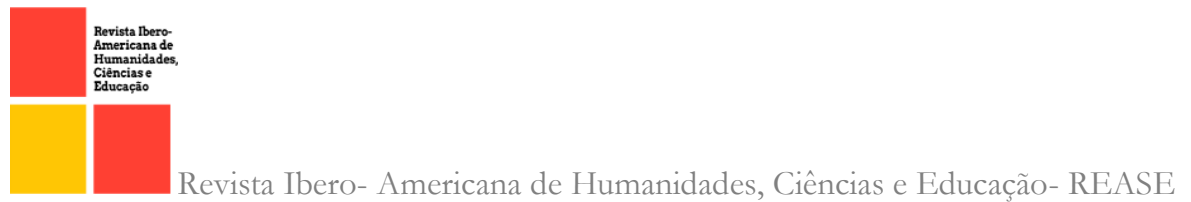

neste grupo pode predizer o desenvolvimento de obesidade na vida adulta. Dentre os profissionais atuantes na Atenção Básica, destaca-se a importância da inserção do nutricionista, uma vez que o enfoque do modelo de saúde proposto pelo governo não é simplesmente de recuperação da saúde e tratamento da doença, mas, principalmente, de ações voltadas para a promoção da saúde e prevenção de agravos. Desta forma, este profissional possui conhecimentos capazes de melhorar o perfil epidemiológico e nutricional por meio da adoção de hábitos alimentares saudáveis pela população. (Layonne de Sousa Carvalho et al., 202I)

A alimentação foi reconhecida como direito na Constituição brasileira em 2oı. E como um dos determinantes da saúde da população na lei que criou o Sistema Único de Saúde (SUS); cabe à direção nacional do SUS a competência de "formular, avaliar e apoiar políticas de alimentação e nutrição (Brasil. Lei No 8.080, de I9 de setembro de 1990)

Enfatizando a importância de práticas alimentares e estilos de vida saudáveis como um componente importante para a promoção da saúde já nos primeiros anos de vida. (SISVAN,2008)

A realização de refeições fora do lar destaca-se como um comportamento que favorece o consumo excessivo de alimentos ultraprocessados e energia, e, consequentemente, o ganho de peso acima do recomendado e o desenvolvimento de Doenças crônicas não transmissíveis (DCNT). (Donin AS et al., 2018)

A má alimentação é fator primordial para o aparecimento das DCNT, dentre elas, a diabetes, neoplasias e dislipidemia. Estudos mostram que uma boa alimentação vem diminuindo a pré-disposição genética das DCNT, aumento da qualidade de vida do indivíduo, evitando o consumo de fontes de alimentos em gorduras saturadas e trans, presentes em fast foods, refrigerantes, frituras e doces.

Dessa forma, o presente estudo tem como objetivo identificar a Importância do Nutricionista na Atenção Primária na Prevenção e Tratamento das DCNT, contribuindo com mais informações para sociedade no planejamento e execução de ações de educação 
alimentar e nutricional, avaliação nutricional, desenvolvimento de programas que diminuam a incidência dessas doenças, estimulando a mudança do estilo de vida.

\section{METODOLOGIA}

Trata-se de uma revisão de literatura narrativa, onde foram utilizados artigos científicos que abordavam a prevenção e tratamento das doenças crônicas não transmissíveis na atenção primária em todo território brasileiro e a importância do profissional nutricionista.

Os artigos utilizados estão indexados nas plataformas de base de dados Medline/Pubmed e SciELO, no período de 2008 a 202I, no idioma português, utilizando os seguintes descritores, de acordo com os Descritores em Saúde (DeCs): nutrição, atenção primária , alimentação. $\mathrm{Na}$ busca foram utilizados os operadores boleanos de pesquisa AND, OR e NOT.

\section{RESULTADOS E DISCURSÃO}

As DCNTs têm grande influência no perfil de saúde pública e desenvolvimento do país, caracterizado pelo crescimento no número de pessoas com sobrepeso ou obesidade. De acordo com a Organização Mundial da Saúde (OMS), o enfretamento da obesidade é um dos mais graves problemas de saúde pública no Brasil e aumentou $67,8 \%$ nos últimos treze anos (ABESO, 2018).

Muitos estudos apontam a transição nutricional como causa, pois o aumento do consumo de alimentos processados e ultraprocessados, ricos em açúcares, gorduras, corantes e sódio e a redução do consumo de alimentos naturais (como frutas e hortaliças) gerou mudanças importantes na estrutura da dieta dos indivíduos, processo que repercute diretamente nas condições atuais de saúde (SANTOS et al., 2019).

Através destes dados, percebemos a importância do profissional nutricionista na atenção primária no sentido de promover saúde, prevenir e tratar doenças crônicas não transmissíveis, evitando seus agravos. Sua atuação efetiva pode trazer muitos benefícios, 

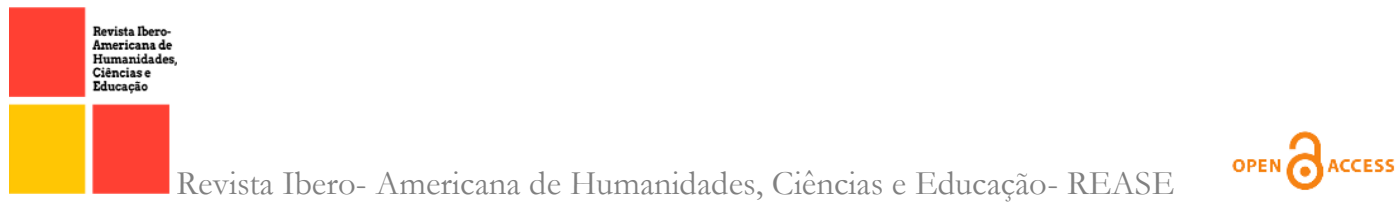

como a redução dos gastos públicos ,pois as despesas com o tratamento das DCNT são responsáveis por grande comprometimento da renda do sistema de saúde. Como mencionado pelo ministério da saúde :Os custos atribuíveis à hipertensão arterial, diabetes e obesidade no Brasil totalizaram R $\$ 3,45$ bilhões em 2018, considerando gastos do SUS com hospitalizações, procedimentos ambulatoriais e medicamentos.(Ministério da saúde,2020). A redução de incapacidades físicas é outro fator de grande interesse público e que interfere diretamente no desenvolvimento econômico do país. Como mencionado no trabalho de Assunção et al.,2020 :As DCNT são incapacitantes, no âmbito da Previdência Social (PS) brasileira, em 2016, as doenças osteomusculares foram a principal causa de afastamentos do trabalho, que justificaram 407 mil auxílios-doença, seguidas das doenças do aparelho digestivo (279 mil), doenças mentais (185 mil), neoplasias (165) e doenças do aparelho circulatório ( $159 \mathrm{mil}$ ) . Além da possível redução das mortes prematuras, Em 2018, no Brasil, foram registrados 720.205 óbitos por DCNT, e, destes, 42,2\% (n=303.776) ocorreram prematuramente, ou seja, entre 30 e 69 anos de idade, com 30r,4 óbitos prematuros a cada ioomil habitantes.(Ministério da saúde,2020)

Ao longo dos últimos anos a alta prevalência da DCNT é um problema globale que o seu controle tem sido um verdadeiro desafio. Como exemplo desse esforço para contenção do avanço das DCNT temos desde o ano de 20II, lançado pelo ministério da saúde o plano de ações estratégicas ,para o enfrentamento das DCNT no Brasil,20II-2022. Nesse plano foram retratadas as quatro principais doenças crônicas (circulatórias , canceres, respiratórias crônicas e diabetes) Entre as 12 metas estabelecidas no plano, temos a redução da mortalidade prematura (30-69 anos) por DCNT em 2\% ao ano.(Ministério da saúde ,2020) Meta essa que foi alcançada como foi visto no estudo realizado por MALTA, D.C. ET AL , onde tinha por objetivo analisar as tendências de mortalidade prematura por DCNT no período de 2000 a 2013 , bem como a probabilidade de morte por essas doenças projetada para 2025. Onde os resultados encontrados foram animadores, pois foi possível identificar uma redução de $2,5 \%$ ao ano na taxa de mortalidade prematura por DCNT no Brasil. 


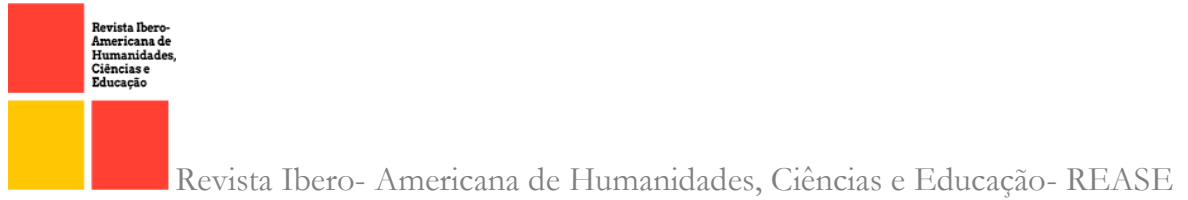

Mota, et. Al 2020, desenvolveu um estudo onde foram entrevistados profissionais e usuários das unidades básicas de saúde, com o objetivo de analisar a importância da inserção do nutricionista em Unidades Básicas de Saúde (UBS), bem como as dificuldades de acesso aos seus serviços a partir da percepção de profissionais e usuários do serviço de saúde. Os usuários entrevistados, com predominância do sexo feminino (82,6\%), onde $56,5 \%$ eram hipertensos, $13,1 \%$ eram diabéticos e hipertensos e 30,4\% relataram não apresentar problemas de saúde.

A partir da análise dos discursos dos usuários, foram identificadas ideias centrais para cada pergunta realizada. Destacou-se que os usuários entrevistados afirmaram que precisavam de orientação nutricional para "melhorar a saúde" (52,2\%), "melhorar a alimentação" (30,4\%), contudo alguns disseram que não precisavam $(17,4 \%)$.

Já os profissionais de saúde, quando questionados se achavam capacitados para oferecer orientações nutricionais à população, 80\% afirmaram que não, demonstrando a necessidade do profissional nutricionista na UBS. A partir do relato dos profissionais de saúde pode-se constatar que existe a necessidade da atuação do nutricionista na APS, visto que os profissionais relataram não ter capacitação adequada e suficiente para orientar os usuários, deixando nítida a relevância do nutricionista e sua contribuição como educador.

Em contra partida, de acordo com o mesmo estudo, as percepções dos usuários apontaram várias dificuldades no acesso ao cuidado nutricional e na sua ampliação como a demora e burocracia para conseguir uma consulta, falta de informação sobre a presença do nutricionista na UBS e aspectos relacionados à gestão tais como a falta do profissional em quantidade suficiente e estrutura física deficiente.

Outro estudo que afirma as dificuldades de acesso, desenvolvido por Pimentel et al., na ESF de cinco municípios brasileiros pontou que os desafios enfrentados para a implementação das ações de alimentação e nutrição são abrangentes, que partem desde questões socioeconômicas e culturais, a falta de integração e articulação entre as equipes, pouco incentivo dos gestores de saúde no campo de infraestrutura, dificuldade de 


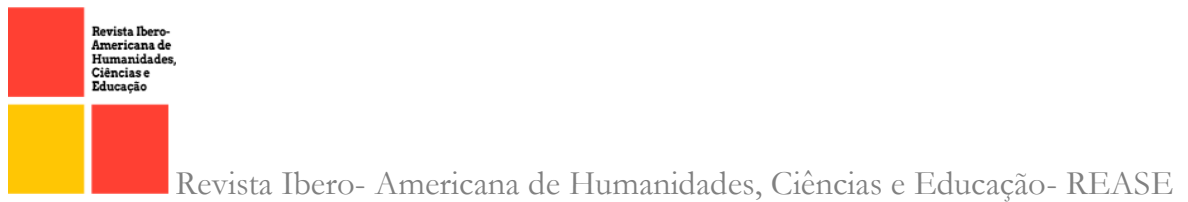

mudanças efetivas nos hábitos inadequados dos indivíduos e a influência negativa da mídia na população quanto ao consumo de alimentos não saudáveis.

Tendo por base que alguns fatores de risco que levam as DCNT, são modificáveis, reforçamos a importância da implementação de ações de alimentação e nutrição, por um profissional qualificado, que a partir da vivencia junto á comunidade ,possa identificar quais a necessidade e problemas enfrentados por aquela população. Traçando metas e objetivos e levando em consideração a diversidade cultural, econômica e social dos usuários.

Em 3i de dezembro de 2019, a Organização Mundial da Saúde foi notificada sobre vários casos de pneumonia na cidade de Wuhan, China. Falava-se sobre uma nova cepa de coronavírus que não tinha sido constatada antes em humanos. A COVID-ı́ é uma doença infecciosa causada pelo novo coronavírus (SARS-CoV-2) e tem como sintomas febre, cansaço, tosse, dificuldade para respirar, perda do paladar, perda do olfato, entre outros. A maioria das pessoas (aproximadamente de 80\%) se recupera da COVID-i9 sem a necessidade de tratamento hospitalar. Pessoas idosas e as que possuem comorbidades, como pressão alta, problemas cardíacos e do pulmão, diabetes ou câncer, têm maior risco de ficarem gravemente doentes. Porém, qualquer pessoa pode pegar a COVID-ı e ficar gravemente doente. (OPAS)

Diante da situação em que o país se encontra, fica cada vez mais necessário a atuação interdisciplinar nas UBSs, incluindo o nutricionista, para também na disseminação de informações seguras sobre DCNTs e COVID-ı, já que a onda de fake News ganhou espaço nos últimos tempos.

\section{CONCLUSÃO}

O aumento nos casos de doenças crônicas não transmissíveis e as mudanças socioeconômicas e comportamentais dos indivíduos é visto pelos órgãos de saúde como um desafio. O trabalho do nutricionista é de suma importância, e quando se trata de prescrição dietética ele é o único profissional capacitado e autorizado para tal atributo. 
O papel do nutricionista consiste em prevenir doenças e suas complicações, tratar a pessoa, recuperar o estado nutricional adequado e promover qualidade de vida, podendo trazer benefícios a curto e longo prazo.

A coleta de dados sobre a percepção dos usuários e profissionais da saúde a respeito da importância do nutricionista é essencial, pois são eles que compõem a estrutura e sentem de fato a experiencia da presença desse profissional fazendo a diferença desde o atendimento primário até seu ambiente cotidiano.

Concluímos que a falta do nutricionista acarreta em assistência incompleta e superficial, já que a alimentação se trata de um fator biopsicossocial. É a partir, principalmente, do estado nutricional que se pode obter um prognostico e fazer um encaminhamento adequado quando necessário. A prevenção pode ainda minimizar gastos públicos proporcionando mais investimento em outras áreas. Faz-se necessário mais atenção dos governos em relação a saúde e pesquisa.

\section{REFERÊNCIAS}

ABESO. Associação Brasileira para o Estudo da Obesidade e da Síndrome Metabólica. Diretrizes Brasileira de Obesidade. Mapa da obesidade, 2018. Disponível em: https://abeso.org.br/obesidade-e-sindrome-metabolica/mapa-da-obesidade/. (acesso em I5 de setembro de 202I)

Brasil. Emenda Constitucional no 64, de 4 de fevereiro de 2010. Altera o art. 6o da Constituição Federal, para introduzir a alimentação como direito social. [Internet]. 2oro. Available from: http:// www.planalto.gov.br/ccivil_03/constituicao/emendas/emc/ emc64.htm

Brasil. Lei No 8.080, de 19 de setembro de 1990. Dispõe sobre as condições para promoção, proteção e recuperação da saúde, a organização e o funcionamento dos serviços correspondentes e dá outras providências. 1990.

Brasil. Ministério da Saúde (MS). Departamento de Atenção Básica/Secretaria de Atenção à Saúde. Proto - colos do Sistema de Vigilância Alimentar e Nutricional SISVAN na assistência à saúde. Brasília: MS; 2008 
De Sousa Carvalho, Layonne, et al. "Desafios do nutricionista no combate à obesidade na atenção primária à saúde no Brasil." International Journal of Development Research II.05 (2021): $47415-47418$.

Donin AS, Nightingale CM, Owen CG, Rudnicka AR, Cook DG, Whincup PH. Takeaway meal consumption and risk markers for coronary heart disease, type 2 diabetes and obesity in children aged 9-Io years: a cross-sectional study. Arch Dis Child 2018; I03:431-6

Folha Informativa COVID-I9-Escritório da OPAS e da OMS no Brasil (20II) https://www.paho.org/pt/covidi9 (consulta I5 de setembro de 202I)

https://bvsms.saude.gov.br/bvs/publicacoes/atlas_situacao_alimentar_nutricional_popula cao_adulta.pdf

Anos de vida perdidos por DCNT atribuídos aos riscos ocupacionais no Brasil: estudo GBD 2016 https://www.scielo.br/j/rsp/a/F6Nws8DBPzKznbnD3kcy3sD/?lang=pt\# https://antigo.saude.gov.br/images/pdf/2020/October/or/Plano-DANT-vers--oConsulta-p--blica.pdf

https://antigo.saude.gov.br/images/pdf/2020/October/oI/Plano-DANT-vers--oConsulta-p--blica.pdf

Mota, Juliana Ramos da., Oliveira, Daniele dos Santos., Santos, Adriana Correia dos., Chagas, Bárbara Lúcia Fonseca., \& Voci, Silvia Maria. RG\&PP, io(2):207-223, 2020. DOI: Io.I1606/rgpp.vioi2.184531.

Pimentel VRM, Sousa MF, Hamann EM, Mendonça AVM. Food and nutrition in the Family Health Strategy in five Brazilian cities. Ciência \& Saúde Coletiva. 2014; 19(I): 4957.

Plano de ações estratégicas para o enfrentamento das doenças crônicas e agravos não transmissíveis no Brasil

SANTOS, D. S. dos. et al. Transição nutricional na adolescência: uma abordagem dos últimos io anos. Revista Eletrônica Acervo Saúde, n. 20, p. e477-e477, 2019. 